\title{
The Inhomogeneous Extended Bose-Hubbard Model: A Mean-Field Theory
}

\author{
Jamshid Moradi Kurdestany \\ Department of Physics, Indian Institute of Science, Bangalore 560 012, India \\ Ramesh V.Pa团 \\ Department of Physics, Goa University, Taleigao Plateau, Goa 403 206, India \\ Rahul Pandit团 \\ Centre for Condensed Matter Theory, Department of Physics, \\ Indian Institute of Science, Bangalore 560012, India.
}

(Dated: September 23, 2018)

\begin{abstract}
We develop an inhomogeneous mean-field theory for the extended Bose-Hubbard model with a quadratic, confining potential. In the absence of this potential, our mean-field theory yields the phase diagram of the homogeneous extended Bose-Hubbard model. This phase diagram shows a superfluid (SF) phase and lobes of Mott-insulator (MI), density-wave (DW), and supersolid (SS) phases in the plane of the chemical potential $\mu$ and on-site repulsion $U$; we present phase diagrams for representative values of $V$, the repulsive energy for bosons on nearest-neighbor sites. We demonstrate that, when the confining potential is present, superfluid and density-wave order parameters are nonuniform; in particular, we obtain, for a few representative values of parameters, spherical shells of SF, MI, DW, and SS phases. We explore the implications of our study for experiments on cold-atom dipolar condensates in optical lattices in a confining potential

PACS numbers: $05.30 \mathrm{Jp}, 67.85 . \mathrm{Hj}, 73.43 \mathrm{Nq}$
\end{abstract}

\section{INTRODUCTION}

Experimental studies of quantum phase transitions [1-4] in systems of cold atoms in traps, with an imposed optical lattice, have led to a renewal of interest in theoretical studies of lattice models of interacting bosons 5 77. Examples of such transitions include one from a superfluid (SF) to a bosonic Mott-insulator (MI). This transition was predicted by mean-field theories, such as those of Refs. [5, 7], and obtained in Monte-Carlo simulations [6] of the Bose-Hubbard model before it was realized experimentally.

In addition to the optical-lattice potential, a confining potential, most often quadratic, is present in all cold-atom experiments. This leads to inhomogeneities in the phases that are obtained: simulations [8, 9] of the Bose-Hubbard model, with such a confining potential, and experiments [10, 11] on interacting bosons in optical lattices, with a confining potential, have both seen alternating shells of SF and MI regions.

Mean-field theories for the Bose-Hubbard model were first developed for the homogeneous case [5, 7, 12]. These theories were then extended to the inhomogeneous case 13] to develop an understanding of the Bose-glass phase in the disordered Bose-Hubbard model. In recent work [14] we have shown how the effects of such a confining potential can be treated, at the level of mean-field theory, as was done in the Bose-glass case [13]; in particular, we have provided a natural framework for understanding alternating SF and MI shells, mentioned above. Here we extend this inhomogeneous mean-field theory to account for the different types of phases, SF, MI, density-wave (DW), and supersolid (SS), which can occur in the extended Bose-Hubbard model 15].

The principal motivation for undertaking such a study of the extended Bose-Hubbard model is provided by the experiments that have obtained a dipolar condensate of ${ }^{52} \mathrm{Cr}$ atoms [16]. To understand these experiments we must study lattice models of bosons with long-range interactions [17] and not merely the Bose-Hubbard model with a repulsive interaction between bosons on the same lattice site. The simplest model that goes beyond such onsite interactions is the extended Bose-Hubbard model, which allows for repulsive interactions between bosons on nearestneighbor sites and the aforementioned onsite interaction. In addition to SF and MI phases of the Bose-Hubbard

\footnotetext{
*Electronic address: jamshid@physics.iisc.ernet.in, jmkurdestany@gmail.com

$\dagger$ Electronic address: rvpai@unigoa.ac.in

${ }_{\ddagger}^{\ddagger}$ Electronic address: rahul@physics.iisc.ernet.in Also at Jawaharlal Nehru Centre For Advanced Scientific Research, Jakkur, Bangalore, India
} 
model, this extended model can have a density wave (DW) phase, in which the mean density of bosons is different on the two sublattices of the hypercubic lattices we consider, and a super-solid (SS) phase (see, e.g., Refs. [15, 18, 19]).

Before we present the details of our study, we summarize our principal results. We first develop a mean-field theory for the homogeneous, extended Bose-Hubbard model by developing on the work of our group on Bose-Hubbard models for the spinless and spin-1 cases [7, 12]; this yields the SF, MI, DW, and SS phases and the transitions between them, which have been studied by a Gutzwiller-type approximation [15] that is akin to, but not the same as, our mean-field theory. We then develop an inhomogeneous mean-field theory for the inhomogeneous extended, Bose-Hubbard model by generalizing our inhomogeneous mean-field theory for the Bose-Hubbard model [14]. In particular, when we use a quadratic confining potential in three dimensions (3D), our theory yields inhomogeneous phases with spherical shells of SF, MI, DW, and SS states. The precise way in which these phases alternate depends on the parameters of the model; we study a few illustrative cases explicitly for which we present order-parameter profiles and their Fourier transforms. We also discuss the experimental implications of our work.

The remaining part of this paper is organized as follows. In Sec. II we introduce the inhomogeneous extended Bose-Hubbard model and then develop an inhomogeneous mean-field theory for it. In Sec. III we present the results of our mean-field theory. Section IV contains concluding remarks; here we give a brief comparison of our work with earlier studies and we explore the experimental implications of our study.

\section{MODEL AND MEAN-FIELD THEORY}

We study the inhomogeneous, extended Bose-Hubbard model that is defined by the Hamiltonian

$$
\begin{aligned}
\frac{\mathcal{H}}{z t} & =-\frac{1}{z} \sum_{<i, j>}\left(a_{i}^{\dagger} a_{j}+h . c\right)+\frac{1}{2} \frac{U}{z t} \sum_{i} \hat{n}_{i}\left(\hat{n}_{i}-1\right) \\
& +\frac{V}{z t} \sum_{<i, j>} \hat{n}_{i} \hat{n}_{j}-\frac{1}{z t} \sum_{i} \mu_{i} \hat{n}_{i},
\end{aligned}
$$

where $t$ is the amplitude for a boson to hop from site $i$ to its nearest-neighbor site $j, z$ is the nearest-neighbor coordination number, $\langle i, j\rangle$ are nearest-neighbor pairs of sites, h.c. denotes the Hermitian conjugate, $a_{i}^{\dagger}, a_{i}$, and $\hat{n}_{i} \equiv a_{i}^{\dagger} a_{i}$ are, respectively, boson creation, annihilation, and number operators at the site $i$, the repulsive potential between bosons on the same site is $U$, the chemical potential $\mu_{i}$ controls the number of bosons at the site $i$, and $V$ is the repulsive interaction between bosons on nearest-neighbor sites. In the inhomogeneous case, the chemical potential is $\mu_{i} \equiv \mu-V_{T} R_{i}^{2}$, where $\mu$ is the uniform part of the chemical potential, $V_{T}$ the strength of the harmonic confining potential, $R_{i}^{2} \equiv \sum_{n=1}^{d} X_{n}^{2}(i)$, where $X_{n}(i), 1 \leq n \leq d$, are the Cartesian coordinates of the site $i$ and $d$ is dimension of the hypercubic lattice (we study $d=3$ explicitly); the origin is chosen to be at the center of this lattice. Clearly, if we set $V=0$, we obtain the inhomogeneous Bose-Hubbard model of Ref. [14], which we follow in our mean-field treatment below. In this paper, we set $z t=1$, i.e., we measure all energies in units of $z t$.

If $t=0$ and $V_{T}=0$, the model (11) exhibits (a) MI phases, which have an integral number of bosons per site, or (b) DW phases, in which bosons preferentially occupy one of the sublattices, say $\mathcal{A}$, of the bipartite, hypercubic lattices we consider; the MI phases are favored at large values of $U$ whereas the DW phases appear if $V$ is large. A variety of DW phases are possible; we denote them by DW $\mathrm{M} / 2$; here $\mathrm{M}$ is the number of atoms per unit cell and 2 denotes that the unit cell is doubled, i.e., the length of its side is 2 . For example, when $t=0$ and $V_{T}=0$, the DW $1 / 2$ phase has 1 boson on sublattice $\mathcal{A}$ and none on sublattice $\mathcal{B}$ (or vice versa); in DW $3 / 2$ there is 1 boson on sublattice $\mathcal{A}$ and 2 on sublattice $\mathcal{B}$ (or vice versa).

If $V_{T}=0$ but $t \neq 0, \mathrm{SF}$ or SS phases can be stabilised because the bosons can hop through the lattice. Nonuniform states appear when we allow $V_{T} \neq 0$ as we show below via our inhomogeneous mean-field theory.

We now generalize the intuitively appealing mean-field theory of Ref. 7], which has been developed for the homogeneous Bose-Hubbard model and then extended to the inhomogeneous case in Refs. [13, 14]. Our generalization introduces order parameters that are capable of distinguishing between DW, SS, SF, and MI phases. Conventional mean-field theories introduce a decoupling scheme that reduces a model with interacting bosons or fermions to an effective, noninteracting problem, which can be solved easily because the effective Hamiltonian is quadratic in boson or fermion operators. By contrast, the mean-field theories of Refs. [7, 14], for the case $V=0$, decouple the hopping term in Eq. (11), which is quadratic in boson operators, to obtain an effective, one-site Hamiltonian, which can be diagonalized numerically. To generalize this to the case $V>0$, we have to decouple the number operators in the extended Bose-Hubbard term in Eq. (1). In particular, we decouple the first and third terms of Eq. (1) to obtain an effective one-site problem, which neglects quadratic deviations from equilibrium values (denoted by angular brackets). 
The two approximations we use are as follows:

$$
\begin{aligned}
& a_{i}^{\dagger} a_{j} \simeq\left\langle a_{i}^{\dagger}\right\rangle a_{j}+a_{i}^{\dagger}\left\langle a_{j}\right\rangle-\left\langle a_{i}^{\dagger}\right\rangle\left\langle a_{j}\right\rangle ; \\
& \hat{n}_{i} \hat{n}_{j} \simeq\left\langle\hat{n}_{i}\right\rangle \hat{n}_{j}+\hat{n}_{i}\left\langle\hat{n}_{j}\right\rangle-\left\langle\hat{n}_{i}\right\rangle\left\langle\hat{n}_{j}\right\rangle ;
\end{aligned}
$$

here the superfluid order parameter and the local density for the site $i$ are, respectively, $\psi_{i} \equiv\left\langle a_{i}\right\rangle$ and $\rho_{i} \equiv\left\langle\hat{n}_{i}\right\rangle$, respectively. The approximation (2) can now be used to write the Hamiltonian (1) as a sum over single-site, mean-field Hamiltonians $\mathcal{H}_{i}^{M F}$ as follows:

$$
\begin{aligned}
\mathcal{H}^{M F} & \equiv \sum_{i} \mathcal{H}_{i}^{M F}, \\
\frac{\mathcal{H}_{i}^{M F}}{z t} & \equiv \frac{1}{2} \frac{U}{z t} \hat{n}_{i}\left(\hat{n}_{i}-1\right)-\frac{\mu_{i}}{z t} \hat{n}_{i}-\left(\phi_{i} a_{i}^{\dagger}+\phi_{i}^{*} a_{i}\right) \\
& +\frac{1}{2}\left(\psi_{i}^{*} \phi_{i}+\psi_{i} \phi_{i}^{*}\right)+\frac{V}{t}\left(\hat{n}_{i} \bar{\rho}_{i}-\rho_{i} \bar{\rho}_{i}\right),
\end{aligned}
$$

where the superscript $M F$ stands for mean field, and $\phi_{i} \equiv \frac{1}{z} \sum_{\delta} \psi_{i+\delta}, \bar{\rho}_{i} \equiv \frac{1}{z} \sum_{\delta} \rho_{i+\delta}$, and $\delta$ labels the $z$ nearest neighbors of the site $i$. This form of the single-site, mean-field Hamiltonian is suitable for the inhomogeneous case with $V_{T}>0$.

For the homogeneous case, we note that the hypercubic lattices we consider are bipartite, i.e., they can be divided into two sublattices $\mathcal{A}$ and $\mathcal{B}$. Each site on the $\mathcal{A}(\mathcal{B})$ sublattice has $z$ nearest neighbors each one of which belongs to the $\mathcal{B}(\mathcal{A})$ sublattice. Thus, if $V_{T}=0, \psi_{i}=\psi_{A}$ and $\rho_{i}=\rho_{A}$ if $i \in \mathcal{A}$ and $\psi_{i}=\psi_{B}$ and $\rho_{i}=\rho_{B}$ if $i \in \mathcal{B}$, whereas $\phi_{i}=\psi_{B}$ and $\bar{\rho}_{i}=\rho_{B}$ if $i \in \mathcal{A}$ and $\phi_{i}=\psi_{A}$ and $\bar{\rho}_{i}=\rho_{A}$ if $i \in \mathcal{B}$. If we require chemical potentials that are conjugate to $\rho_{A}$ and $\rho_{B}$, respectively, we can introduce $\mu_{i}=\mu_{A}$ if $i \in \mathcal{A}$ and $\mu_{i}=\mu_{B}$ if $i \in \mathcal{B}$; similarly, we can define creation, annihilation, and number operators for each sublattice and hence write the mean-field Hamiltonian (3) as follows:

$$
\begin{gathered}
\mathcal{H}_{\mathcal{A B}}^{\mathcal{M F}} \equiv \mathcal{H}_{\mathcal{A}}^{\mathcal{M F}}+\mathcal{H}_{\mathcal{B}}^{\mathcal{M F}} ; \\
\frac{\mathcal{H}_{A}^{M F}}{z t} \equiv-\left(a_{A} \psi_{B}^{*}+a_{A}^{\dagger} \psi_{B}\right)+\frac{1}{2}\left(\psi_{A} \psi_{B}^{*}+\psi_{A}^{*} \psi_{B}\right) \\
+\frac{V}{t}\left(\hat{n}_{A} \rho_{B}-\rho_{A} \rho_{B}\right)+\frac{1}{2} \frac{U}{z t} \hat{n}_{A}\left(\hat{n}_{A}-1\right)-\frac{\mu_{A}}{z t} \hat{n}_{A} ; \\
\frac{\mathcal{H}_{B}^{M F}}{z t} \equiv-\left(a_{B} \psi_{A}^{*}+a_{B}^{\dagger} \psi_{A}\right)+\frac{1}{2}\left(\psi_{B} \psi_{A}^{*}+\psi_{B}^{*} \psi_{A}\right) \\
+\frac{V}{t}\left(\hat{n}_{B} \rho_{A}-\rho_{B} \rho_{A}\right)+\frac{1}{2} \frac{U}{z t} \hat{n}_{B}\left(\hat{n}_{B}-1\right)-\frac{\mu_{B}}{z t} \hat{n}_{B} .
\end{gathered}
$$

If $V_{T}>0$, we first obtain the matrix elements of $\mathcal{H}_{i}^{M F}$ in the onsite, occupation-number basis $\left\{\left|n_{i}\right\rangle\right\}$, truncated in practice by choosing a finite value for $n_{\max }$, the total number of bosons per site, for a given initial set of values for $\left\{\psi_{i}, \rho_{i}\right\}$. The smaller the values of $U$ and $V$ and the larger the value of $\mu$ the larger must be the value of $n_{\max }$; for the values of $U, V$, and $\mu$ we consider $n_{\max }=6$ suffices; we have checked this in representative cases by carrying out calculations with $n_{\max }=10$. We then diagonalize this matrix, which depends not just on $\psi_{i}$ and $\psi_{i+\delta}$, but also on $\rho_{i}$ and $\rho_{i+\delta}$, to obtain the lowest energy and the corresponding wave function, denoted, respectively, by $E_{g}^{i}\left(\psi_{i}, \psi_{i+\delta} ; \rho_{i}, \rho_{i+\delta}\right)$ and $\Psi_{g}\left(\left\{\psi_{i}\right\},\left\{\rho_{i}\right\}\right)$; from these we obtain the new order parameters $\psi_{i}=\left\langle\Psi_{g}\left(\left\{\psi_{i}\right\},\left\{\rho_{i}\right\}\right)\right|$ $a_{i}\left|\Psi_{g}\left(\left\{\psi_{i}\right\},\left\{\rho_{i}\right\}\right)\right\rangle$ and $\rho_{i}=\left\langle\Psi_{g}\left(\left\{\psi_{i}\right\},\left\{\rho_{i}\right\}\right)\left|\hat{n}_{i}\right| \Psi_{g}\left(\left\{\psi_{i}\right\},\left\{\rho_{i}\right\}\right)\right\rangle$. We then use these new values of $\psi_{i}$ and $\rho_{i}$ as inputs to reconstruct $\mathcal{H}_{i}^{M F}$ and repeat the diagonalization procedure until we achieve self consistency of input and output values to obtain the equilibrium values $\psi_{i}^{e q}$ and $\rho_{i}^{e q}$ (we suppress the superscript $e q$ hereafter for notational convenience). [This self-consistency procedure is equivalent to a minimization of the total energy $E_{g}\left(\left\{\psi_{i}\right\},\left\{\rho_{i}\right\}\right) \equiv$ $\sum_{i} E_{g}^{i}\left(\psi_{i}, \psi_{i+\delta} ; \rho_{i}, \rho_{i+\delta}\right)$ with respect to $\psi_{i}$ and $\rho_{i}$.] Given the form of the confining potential, the self-consistent solutions for $\left\{\psi_{i}, \rho_{i}\right\}$ must have spherical (circular) symmetry in the three-dimensional (two-dimensional) case; we use this spherical symmetry in obtaining the self-consistent solutions. If $V_{T}=0$, we only need the four order parameters $\psi_{A}, \psi_{B}, \rho_{A}$, and $\rho_{B}$ so the problem of finding self-consistency solutions is much simpler than it is in the inhomogeneous case with $V_{T}>0$. In principle, $\left\{\psi_{i}\right\}$ can be complex, but we find, as in earlier calculations [7, 12 14], that the equilibrium solution is such that $\left\{\psi_{i}\right\}$ are real. 


\section{RESULTS}

In this Section we present the results of the inhomogeneous mean-field theory that we have developed in the previous Section for the extended Bose-Hubbard model (1). We expect that the onsite repulsion between bosons is stronger than the repulsive interaction between bosons on nearest-neighbor sites, so we restrict ourselves to $V<U$. We begin with phase diagrams for the homogeneous case with $V_{T}=0$. We then investigate order-parameter profiles in the presence of the confining potential, i.e., when $V_{T}>0$.

In Figs. 1 (a) and (b), we present phase diagrams in the $(\mu, U)$ plane for the extended Bose-Hubbard model (1), with $V_{T}=0$ and (a) $V / U=0.6$ and (b) $V / U=0.4$, with SF (gray), SS (red), MI (black), and DW (green) phases; the MI phases MI1 and MI2 have, respectively, one and two bosons per site; and DW $1 / 2$ and DW 3/2 are, respectively, DW phases with one and three bosons per unit cell with side 2 ; we take the lattice spacing of the underlying hypercubic lattice to be 1 . The SF phase is favored at small values of $U$. If we hold $\mu$ fixed at low values and increase $U$, the system first undergoes a transition to an SS phase and then to the DW 1/2 phase. The lobe of the MI1 phase appears above the DW 1/2 lobe and the encompassing sliver of the SS phase; the next few DW and MI lobes appear as shown in Figs. 1 (a) and (b). Note that the red slivers of the SS phases encompass the DW lobes completely. Furthermore, the DW and SS phases grow at the expense of the SF and MI phases as $V / U$ increases, as we expect for the extended Bose-Hubbard model (1). The phase diagrams of Figs. 1 (a) and (b) are qualitatively similar to those obtained by a Gutzwiller approximation in Ref. [15].

We obtain these phase diagrams by monitoring the dependence of the SF and DW order parameters on $U$, $V$, and $\mu$. In Fig. 2 we show representative plots of $\psi_{a}$ (red dashed line) and $\psi_{b}$ (black full line), on sublattices $\mathcal{A}$ and $\mathcal{B}$, respectively, versus $\mu$ for $U=12, V_{T}=0, z t=1$, and (a) $V / U=0.6$ and (b) $V / U=0.4$. We also show representative plots of $\rho_{a}$ (red dashed line) and $\rho_{b}$ (black full line), on sublattices $\mathcal{A}$ and $\mathcal{B}$, respectively, versus $\mu$ for $U=12, z t=1$, and (c) $V / U=0.6$ and (d) $V / U=0.4$. We can distinguish these phases from each other by noting the following: in the SF phase $\psi_{A}=\psi_{B}>0$ and $\rho_{A}=\rho_{B}$; in the SS phases $\psi_{A}, \psi_{B}>0, \psi_{A} \neq \psi_{B}$, and $\rho_{A} \neq \rho_{B}$; in the DW phases $\psi_{A}=\psi_{B}=0$ but $\rho_{A} \neq \rho_{B}$; in the MI phases $\psi_{A}=\psi_{B}=0$ and $\rho_{A}=\rho_{B}=m$, a positive integer.
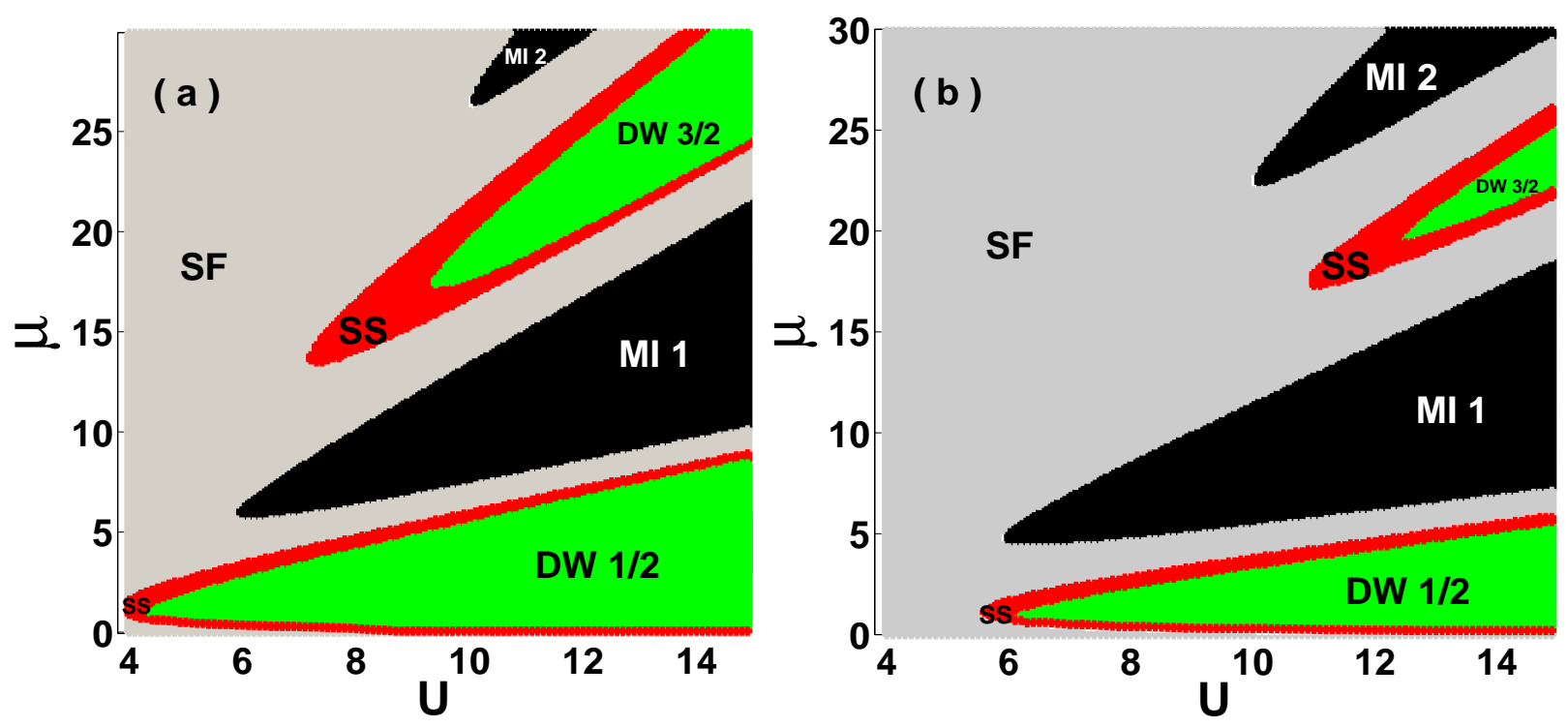

FIG. 1: (Color online) Phase diagrams in the $(\mu, U)$ plane for the extended Bose-Hubbard model with $V_{T}=0$ and $(\mathrm{a}) V / U=0.6$ and (b) $V / U=0.4$ showing SF (gray), SS (red), MI (black), and DW (green) phases. MI1 and MI2 denote, respectively, MI phases with one and two bosons per site; DW $1 / 2$ and DW $3 / 2$ are, respectively, DW phases with one and three bosons per cubic unit cell with side 2 ; the basic lattice spacing is taken to be 1 ; and $z t=1$.

We now use the inhomogeneous mean-field theory, which we have developed in the previous Section, to obtain alternating spherical shells of MI, SF, DW, and SS phases in the 3D, extended Bose-Hubbard model (1) with a quadratic confining potential. We do this by obtaining the order-parameter profiles $\left\{\psi_{i}, \rho_{i}\right\}$ and also by obtaining in-trap density distributions of bosons at different values of $U, V$, and $\mu$.

In particular, we use a 3D simple-cubic lattice with $256^{3}$ sites and $V_{T} /(z t)=0.002$; and we study the following two representative case: (a) $\mu /(z t)=30$ and $V / U=0.6$; and (b) $\mu /(z t)=19$ and $V / U=0.4$. With these parameters the 

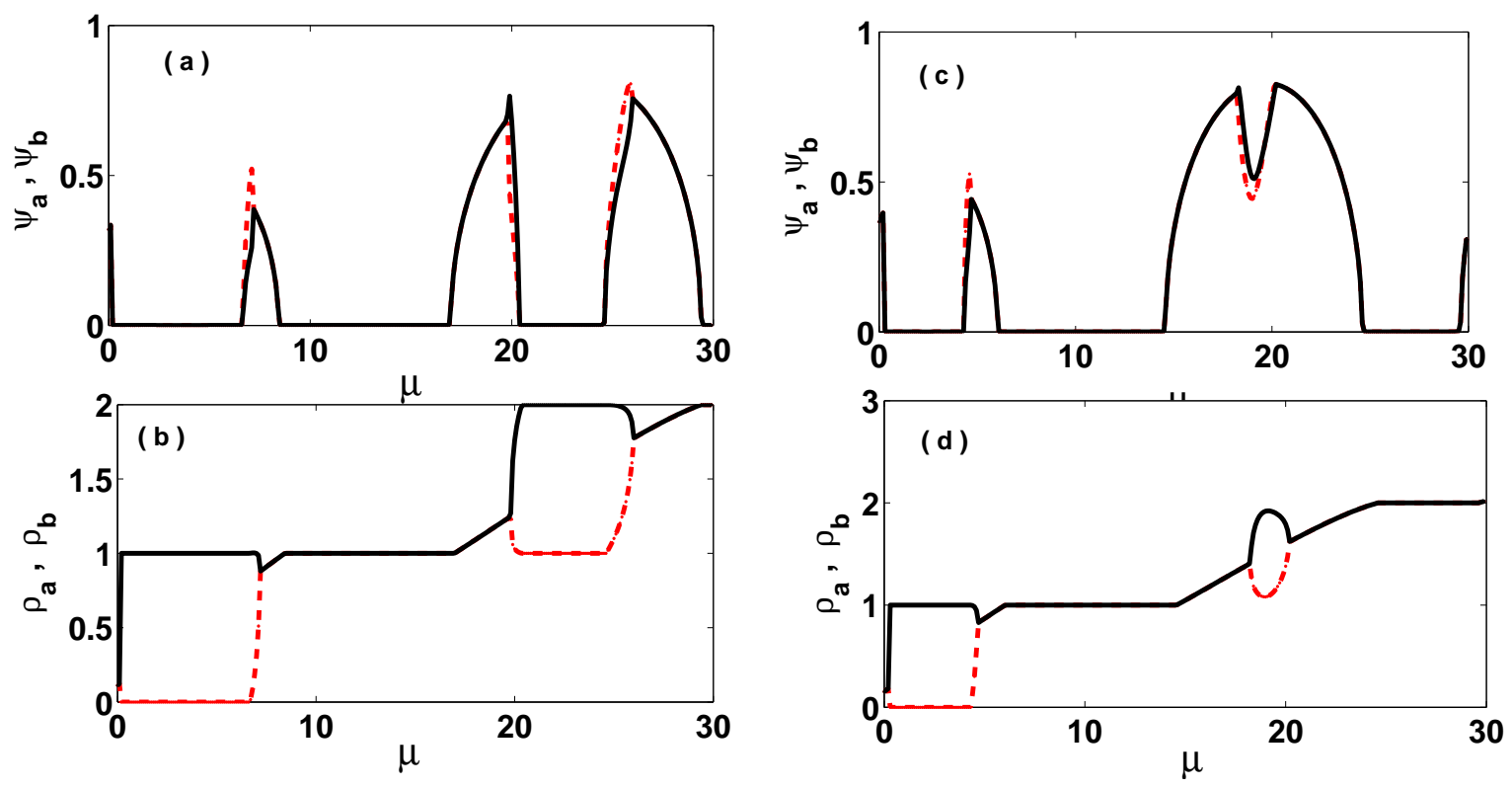

FIG. 2: (Color online) Plots of the superfluid order parameters $\psi_{a}$ (red dashed line) and $\psi_{b}$ (black full line), on sublattices $\mathcal{A}$ and $\mathcal{B}$, versus $\mu /(z t)$ for $U /(z t)=12, V_{T}=0$ and (a) $V / U=0.6$ and (c) $V / U=0.4$. Plots of the density-wave order parameters $\rho_{a}$ (red dashed line) and $\rho_{b}$ (black full line), on sublattices $\mathcal{A}$ and $\mathcal{B}$, versus $\mu /(z t)$ for $U /(z t)=12$ and (b) $V / U=0.6$ and $(\mathrm{d})$ $V / U=0.4$.
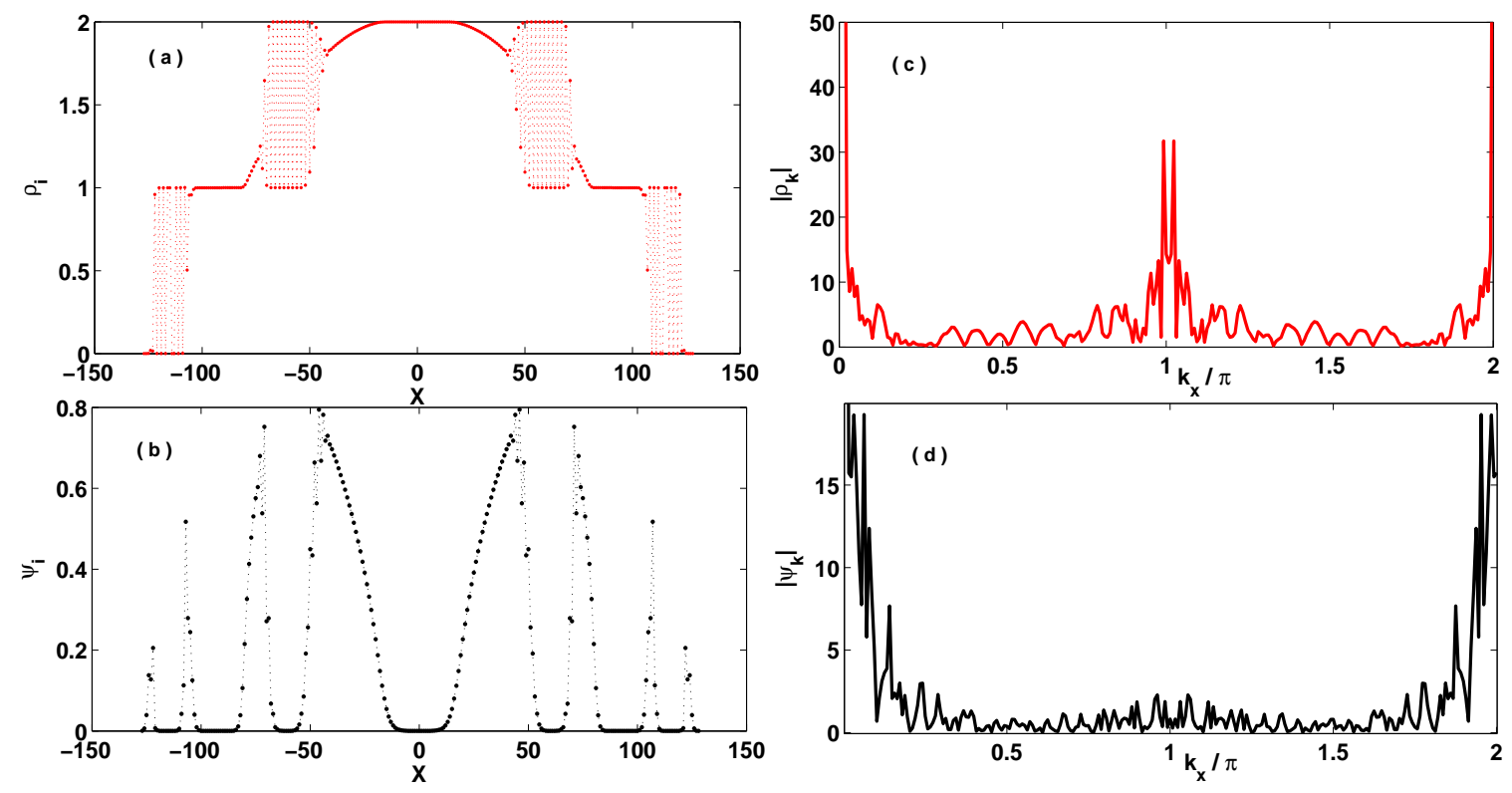

FIG. 3: (Color online) Plots of (a) $\rho_{i}$ (red dashed line and points) and (b) $\psi_{i}$ (black dashed line and points) versus the position $X$ along the line $Y=Z=0$ for $V_{T} /(z t)=0.002, U /(z t)=12, V / U=0.6$ and $\mu /(z t)=30$; the moduli of the one-dimensional Fourier transforms, namely, $\left|\rho_{k}\right|$ and $\left|\psi_{k}\right|$, of the plots in (a) and (b) are plotted, respectively, in (c) and (d) versus the wave vector $k_{X} / \pi$.

total number of bosons $N_{T} \simeq 10^{6}$, which is comparable to experimental values. Furthermore, this choice of parameters leads not only to SF shells and two well-developed MI shells (MI1 and MI2) but also to two well-developed DW shells (DW $1 / 2$ and DW 3/2) and SS shells.

Before we study this shell structure let us explore some order-parameter profiles. Plots of the order parameters $\rho_{i}$ (red dashed line and points) and $\psi_{i}$ (black dashed line and points) versus the position $X$ along the line $Y=Z=0$ are shown in Figs. 3 (a) and (b), respectively, for $V_{T} /(z t)=0.002, U /(z t)=12, V / U=0.6$, and $\mu /(z t)=30$. These 

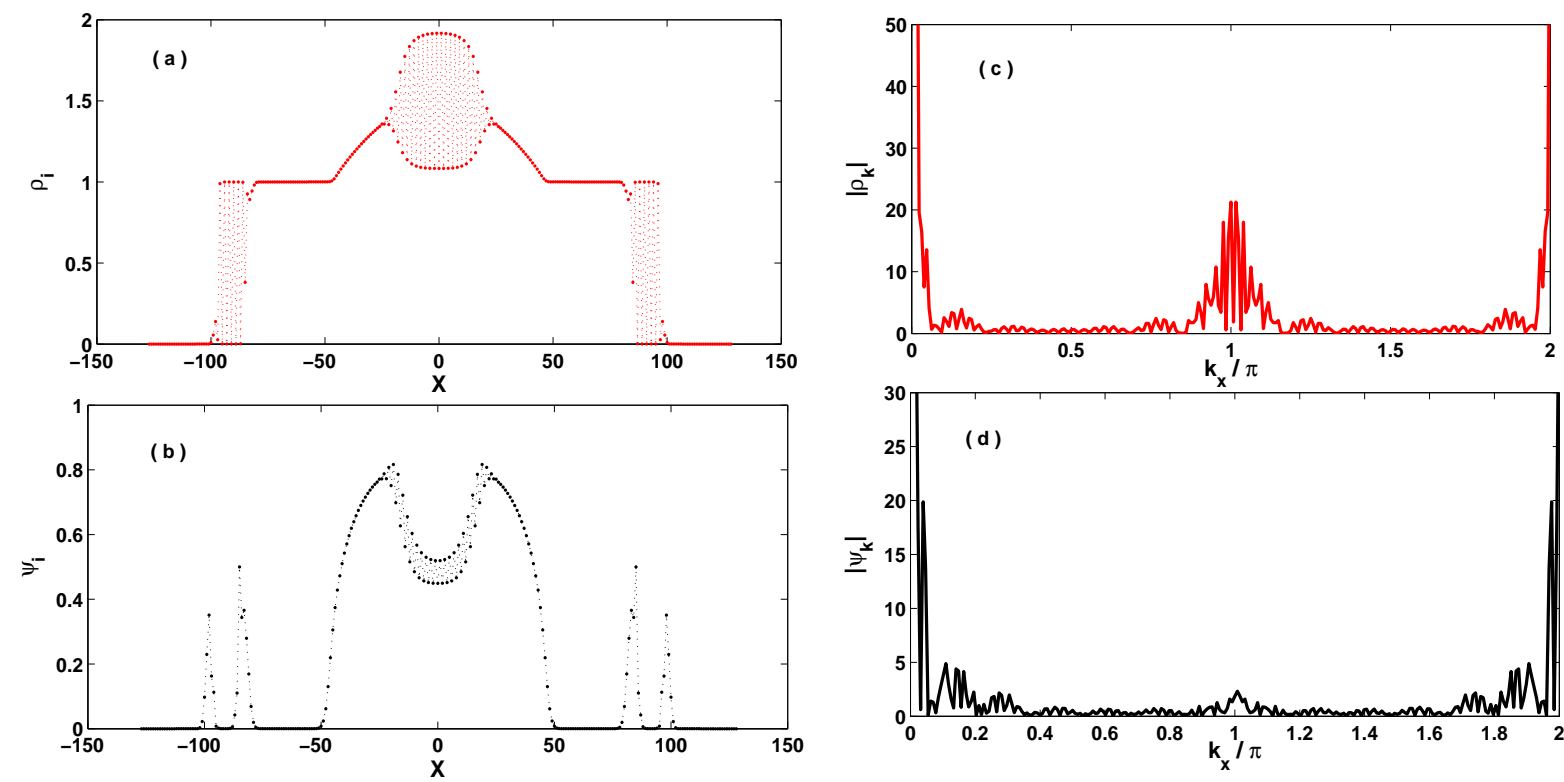

FIG. 4: (Color online) Plots of (a) $\rho_{i}$ (red dashed line and points) and (b) $\psi_{i}$ (black dashed line and points) versus the position $X$ along the line $Y=Z=0$ for $V_{T} /(z t)=0.002, U /(z t)=12, V / U=0.4$ and $\mu /(z t)=19$; the moduli of the one-dimensional Fourier transforms, namely, $\left|\rho_{k}\right|$ and $\left|\psi_{k}\right|$, of the plots in (a) and (b) are plotted, respectively, in (c) and (d) versus the wave vector $k_{X} / \pi$.
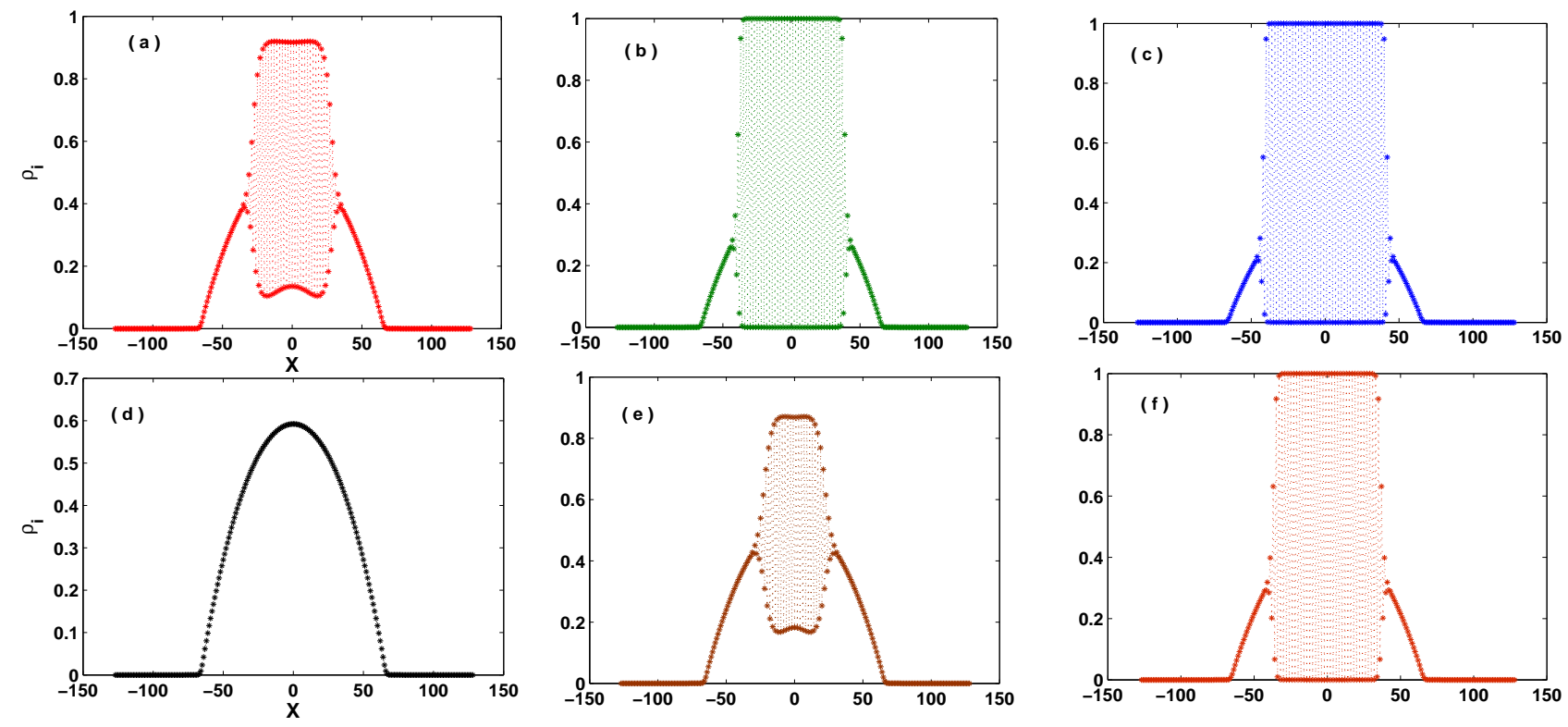

FIG. 5: (Color online) Plots of $\rho_{i}$ versus $X$, along the line $Y=Z=0$, with $\mu /(z t)=1.2, V_{T} /(z t)=0.0005$, and (a) $U /(z t)=4.2$ and $V / U=0.6$, (b) $U /(z t)=5.2$ and $V / U=0.6$, (c) $U /(z t)=6.2$ and $V / U=0.6$, (d) $U /(z t)=4.9$ and $V / U=0.4,(\mathrm{e})$ $U /(z t)=5.9$ and $V / U=0.4$, and (f) $U /(z t)=6.9$ and $V / U=0.4$.

plots show that the region near $X=0$ is an MI2 phase with $\rho_{i}=2$ and $\psi_{i}=0$. As we move outwards from here (either towards $X>0$ or $X<0$ ), we emerge into an SF phase with a noninteger value of $\rho_{i}$ and $\psi_{i}>0$; note that $\rho_{i}$ and $\psi_{i}$ do not oscillate here as functions of $X$. At slightly larger values of $|X|$ the system moves into a very narrow SS region in which both $\rho_{i}$ and $\psi_{i}$ are oscillating functions of $X$. If we increase $|X|$, this SS phase gives way to a DW $3 / 2$ phase in which $\rho_{i}$ oscillates as a function of $X$ but $\psi_{i}=0$. A further increase in $|X|$ yields another very narrow region of the SS phase; this is then followed by a narrow SF region. As we increase $|X|$ some more, the MI1 phase is stabilized; this is followed by a very narrow SF region, which is, in turn, followed by a narrow SS region, and then 

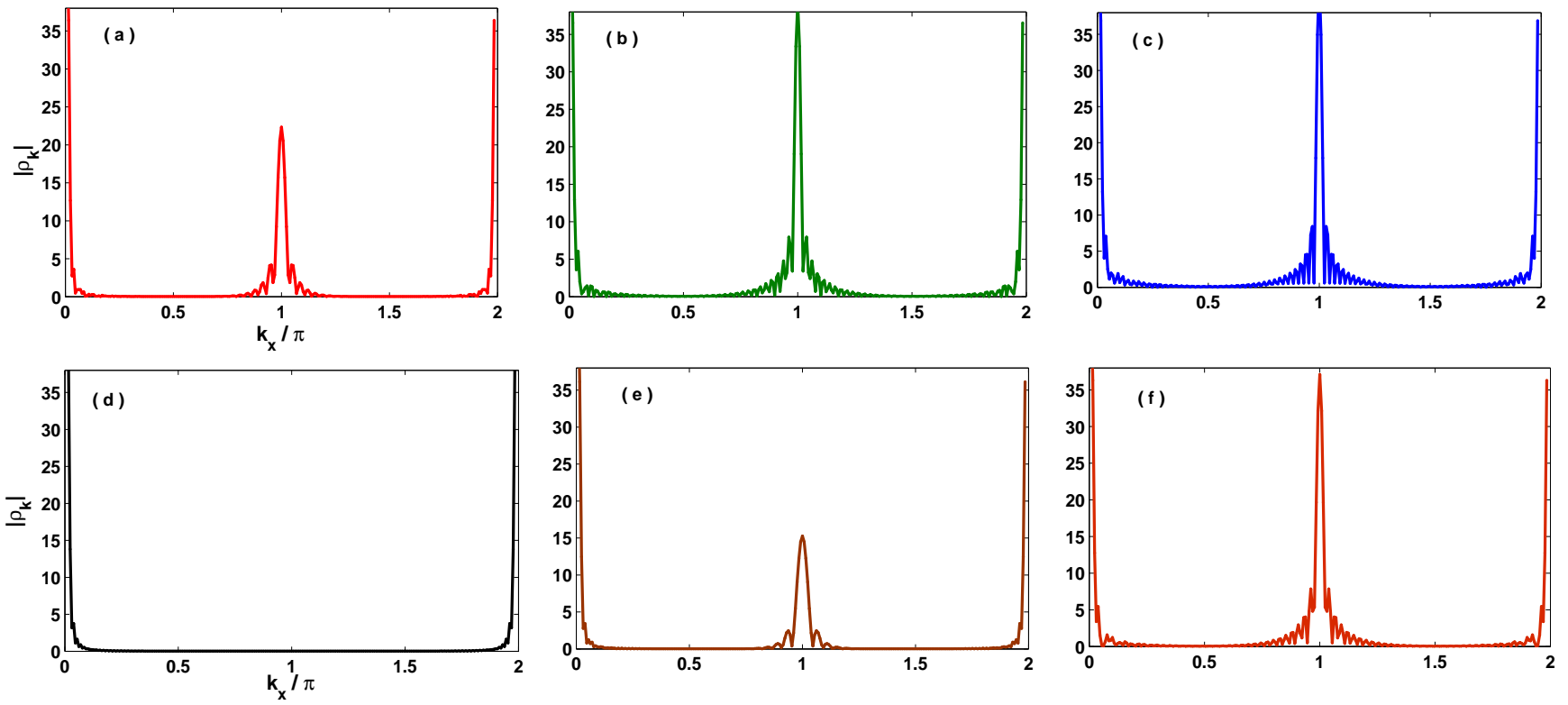

FIG. 6: (Color online) The moduli of the one-dimensional Fourier transforms, namely, $\left|\rho_{k}\right|$, of the plots of $\rho_{i}$ in Figs. 5(a), (b), (c), (d), (e), and (f) are plotted, respectively, in (a),(b), (c), (d), (e), and (f) here versus the wave vector $k_{X} / \pi$.
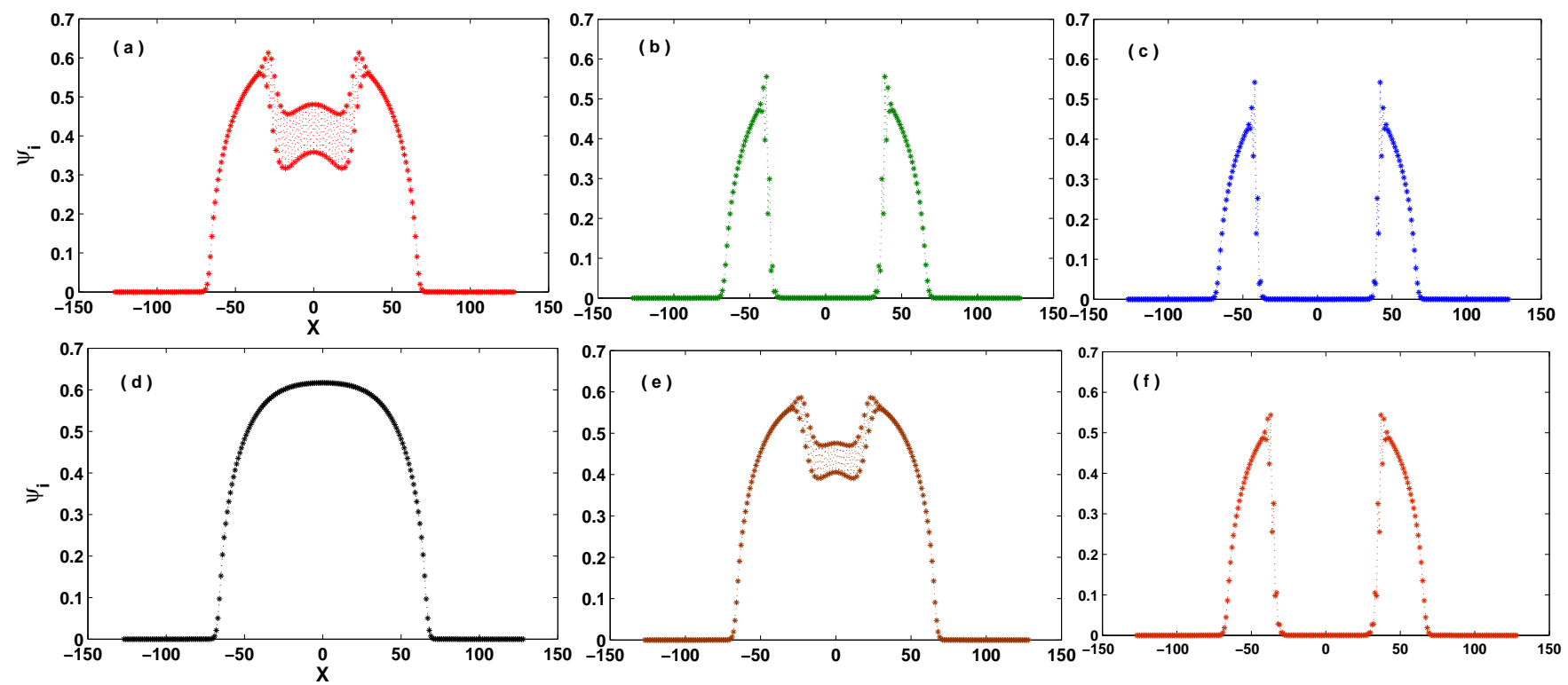

FIG. 7: (Color online) Plots of $\psi_{i}$ versus $X$, along the line $Y=Z=0$, with $\mu /(z t)=1.2, V_{T} /(z t)=0.0005$, and (a) $U /(z t)=4.2$ and $V / U=0.6$, (b) $U /(z t)=5.2$ and $V / U=0.6$, (c) $U /(z t)=6.2$ and $V / U=0.6$, (d) $U /(z t)=4.9$ and $V / U=0.4,(\mathrm{e})$ $U /(z t)=5.9$ and $V / U=0.4$, and (f) $U /(z t)=6.9$ and $V / U=0.4$.

a DW 1/2 regime. This gives way to a very narrow SS region, as $|X|$ increases even more; finally we enter a small region in which the boson density vanishes. Such profiles of $\rho_{i}$ and $\psi_{i}$ imply the shell structure that we explore below.

It is also useful to obtain a complementary, Fourier-representation picture of the profiles in Figs. 3 (a) and (b), because it might be possible to obtain them in time-of-flight measurements (see, e.g., Eq. (44) in Ref. 1]). Threedimensional transforms of the shell structure can be obtained, but they are not easy to visualize; therefore, we present the one-dimensional Fourier transforms of $\rho_{i}(X, Y=0, Z=0)$ and $\psi_{i}(X, Y=0, Z=0)$ with respect to $X$. The moduli of these transforms, namely, $\left|\rho_{k}\right|$ and $\left|\psi_{k}\right|$, of the profiles in Figs. 3 (a) and (b) are plotted, respectively, in Figs. [3 (c) and (d) versus the wave vector $k_{X} / \pi$. The principal peaks in these transforms occur at $k_{X}=0$ (or $2 \pi$ ) and $k_{X}=\pi$; the former is associated with the uniform MI and SF phases; and the latter arises from DW and SS phases 

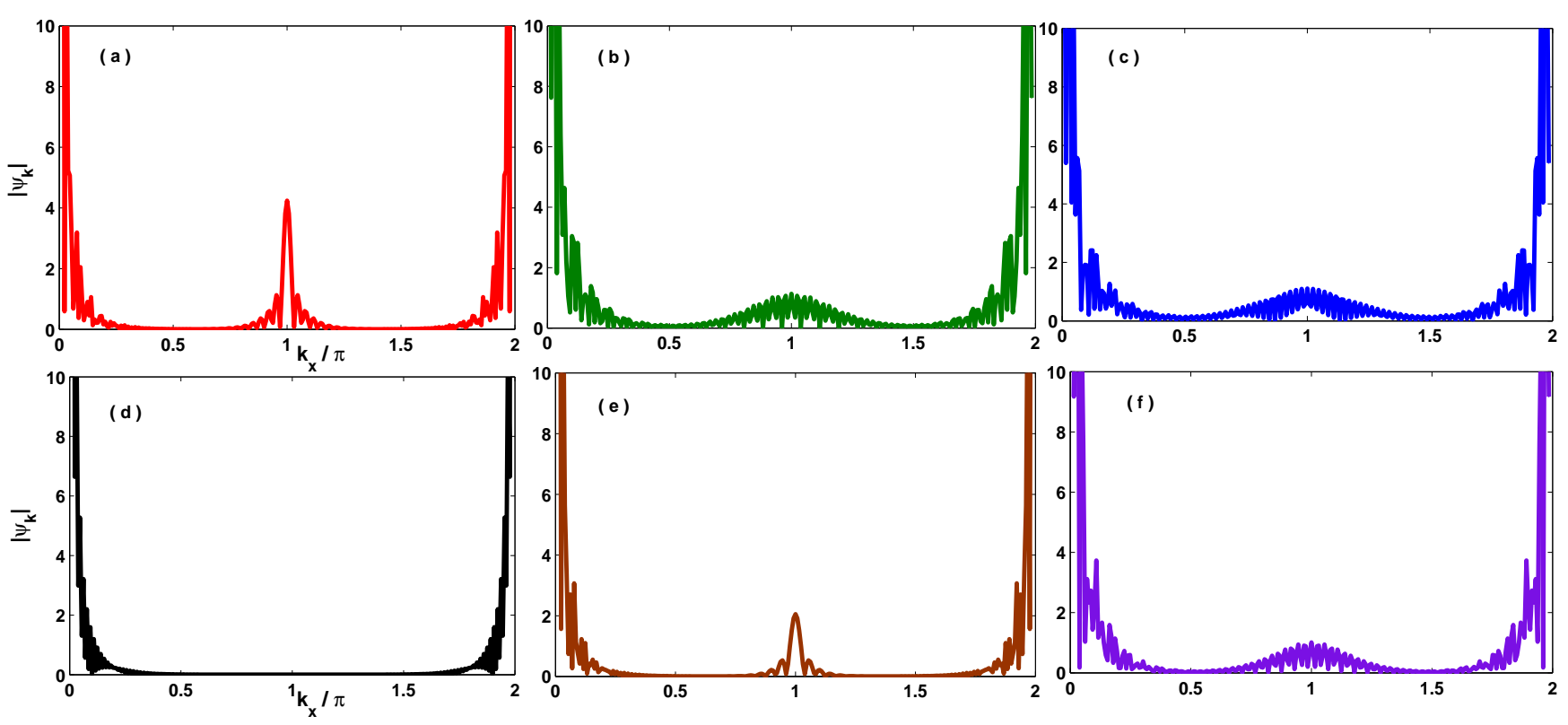

FIG. 8: (Color online) The moduli of the one-dimensional Fourier transforms, namely, $\left|\psi_{k}\right|$, of the plots of $\psi_{i}$ in Figs. 7 (a), (b), (c), (d), (e), and (f) are plotted, respectively, in (a),(b), (c), (d), (e), and (f) here versus the wave vector $k_{X} / \pi$.

in which real-space profiles oscillate as explained above. In an infinite system with no confining potential, these are the only peaks; however, as we have seen above, the quadratic confining potential leads to shells of MI, SF, SS, and DW phases; this shell structure leads to the subsidiary peaks that appear in Figs. 3 (c) and (d) away from $k_{X}=0, \pi$ and $2 \pi$.

Analogs of the order-parameter profiles of Figs. 3 (a) and (b) are given in Figs. (4)(a) and (b), for $\rho_{i}$ (red dashed line and points) and $\psi_{i}$ (black dashed line and points), respectively, versus the position $X$ along the line $Y=Z=0$ for $V_{T} /(z t)=0.002, U /(z t)=12, V / U=0.4$ and $\mu /(z t)=19$. From these plots we see that, in this case, the sequence of phases is SS, SF, MI1, SF, a narrow strip of SS, then DW 1/2, another narrow sliver of SS, and finally a region with vanishing boson density. The moduli of the one-dimensional Fourier transforms, namely, $\left|\rho_{k}\right|$ and $\left|\psi_{k}\right|$, of the plots in Figs. 团 (a) and (b) are plotted, respectively, in Figs. 4 (c) and (d) versus the wave vector $k_{X} / \pi$.

From the profiles in Figs. 3 (a) and (b) and Figs. 4 (a) and (b) it is clear that the precise sequence of MI, SF, SS, and DW shells depends on the parameters in the extended Bose-Hubbard model (1). We illustrate this for other sets of parameter values via representative plots, in Figs. 5 (a)-(f), of the density order parameter $\rho_{i}$ versus $X$, along the line $Y=Z=0$, with $\mu /(z t)=1.2, V_{T} /(z t)=0.0005$, and (a) $U /(z t)=4.2$ and $V / U=0.6$, (b) $U /(z t)=5.2$ and $V / U=0.6$, (c) $U /(z t)=6.2$ and $V / U=0.6$, (d) $U /(z t)=4.9$ and $V / U=0.4$, (e) $U /(z t)=5.9$ and $V / U=0.4$, and (f) $U /(z t)=6.9$ and $V / U=0.4$, respectively. The moduli of the one-dimensional Fourier transforms, namely, $\left|\rho_{k}\right|$, of the plots of $\rho_{i}$ in Figs. 5 (a), (b), (c), (d), (e), and (f) are plotted, respectively, in Figs. 6 (a),(b), (c), (d), (e), and (f) versus the wave vector $k_{X} / \pi$. The corresponding real-space plots of $\psi_{i}$ and the Fourier-space plots of $\left|\psi_{k}\right|$ are given, respectively, in Figs. 7 and 8 ,

These SF, MI, DW, and SS shells appear as annuli 14 in a two-dimensional (2D) planar section $\mathcal{P}_{Z}$ through the 3D lattice, at a vertical distance $Z$ from the center as shown, for $\mathcal{P}_{Z=0}, U /(z t)=12$, and $V_{T} /(z t)=0.002$, in Fig. 9 (a), with $V / U=0.6$ and $\mu /(z t)=30$, and Fig. 9 (b), with $V / U=0.4$ and $\mu /(z t)=19$. In the former case, the core region near $X=Y=Z=0$, has an MI2 phase, whereas, in the latter case, this central region is an SS phase. As we move radially outward from the center, shells of other phases appear; the sequence of shells in Fig. 9 (a) is the one that results from the order-parameter profiles in Figs. 3 (a) and (b); and the sequence of shells in Fig. 9 (b) follows from the profiles in Figs. 团(a) and (b).

For any $2 \mathrm{D}$ planar section $\mathcal{P}_{Z}$ we can calculate integrated, in-trap density profiles such as $N_{m}(Z)$, the number of bosons in the $\rho=m$ MI annuli; similarly, we can calculate $N_{p / q}(Z)$ in the $\rho=p / q$ DW annuli. [Here $m, p$, and $q$ are intergers; e.g., we study $m=1$ or $m=2$ and $p / q=1 / 2$ and $p / q=3 / 2$.] We can also calculate the remaining number of bosons, e.g., $N_{m}^{r}(Z)=N_{T}-N_{m}(Z)$. For the parameter values of Figs. 9 (a) and (b), illustrative integrated, in-trap density profiles are plotted versus $Z$ in Figs. 9 (c) and (d), respectively. These in-trap profiles show the total number of bosons $N_{T}$ (light blue full lines), the number of bosons in MI2 and MI1 regions, $N_{2}$ (black line in Fig. 9 (c)) and $N_{1}$ (brown dash-dotted lines), respectively, the numbers of bosons in DW 3/2 (light green line in Fig. 9 (c)) 
and DW 1/2 (dark green line in Fig. 9 (d)) regions, the numbers of bosons in SS regions (red dashed line), $\left[N_{T}-N_{2}\right]$ (white full line in Fig. 9 (c)), and $\left[N_{T}-N_{1}\right]$ (blue dashed lines). The outermost gray regions contain no bosons. Such integrated, in-trap density profiles have been obtained experimentally in Ref. [10] (see, e.g., their Fig. (3)) for cold-atom systems with SF and MI phases; therefore, it should be possible to carry out similar experiments on the dipolar systems [16, 17] that have motivated our study.
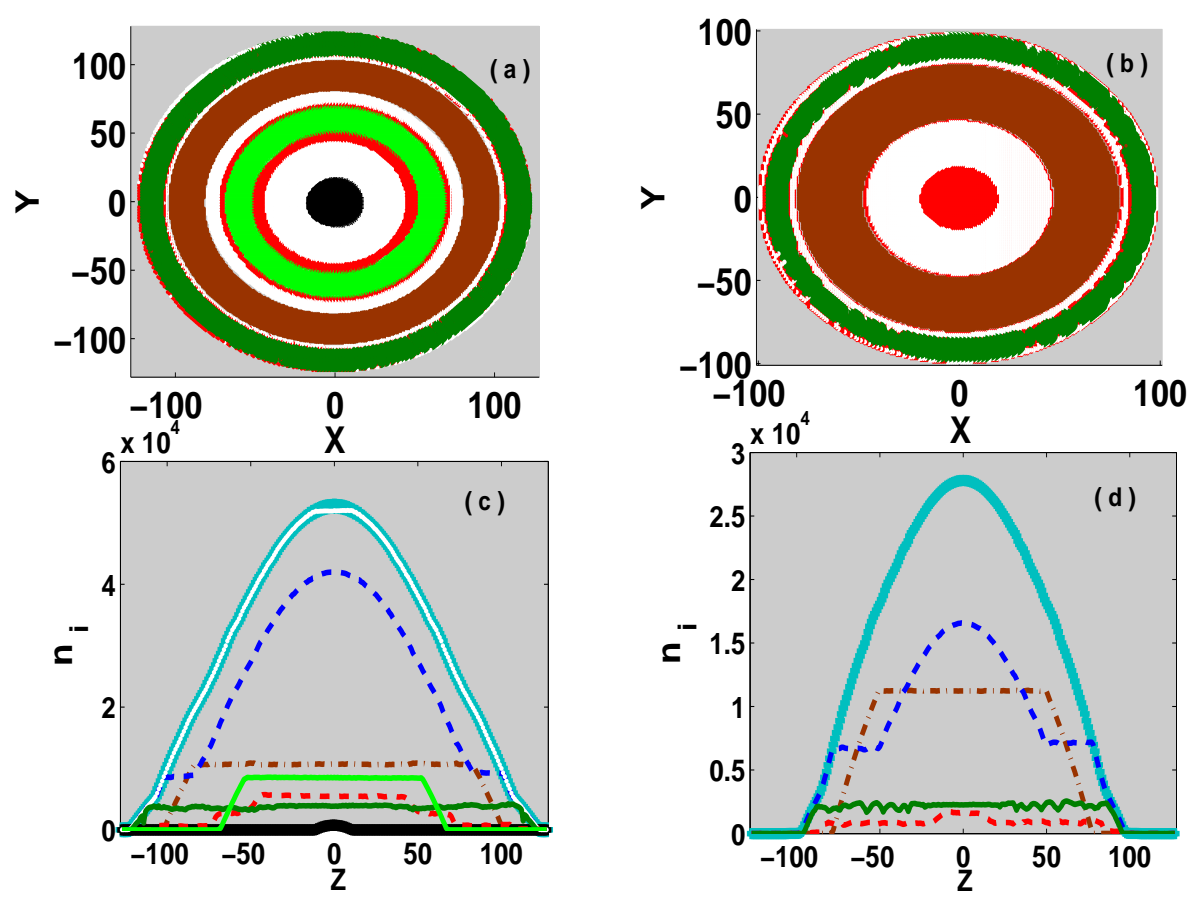

FIG. 9: (Color online) SF (white), MI2 (black), MI1 (brown), SS (red), DW 3/2 (light green), and DW 1/2 (dark green) annuli in the $2 \mathrm{D}$ planar section $\mathcal{P}_{Z=0}$ (see text) with $U /(z t)=12$ and $V_{T} /(z t)=0.002$ and (a) $V / U=0.6$ and $\mu /(z t)=30$ and (b) $V / U=0.4$ and $\mu /(z t)=19$; for these parameter values, the integrated, in-trap density profiles are plotted versus $Z$ in (c) and (d), respectively. These in-trap profiles show the total number of bosons $N_{T}$ (light blue full lines), the number of bosons in MI2 and MI1 regions, $N_{2}$ (black line in (c)) and $N_{1}$ (brown dash-dotted lines), respectively, the numbers of bosons in DW $3 / 2$ (light green line in (c)) and DW 1/2 (dark green line in (d)) regions, the numbers of bosons in SS regions (red dashed line), $\left[N_{T}-N_{2}\right]$ (white full line in (c)), and $\left[N_{T}-N_{1}\right]$ (blue dashed lines) in (c) and (d). The outermost gray regions contain no bosons.

\section{CONCLUSIONS}

We have developed an inhomogeneous mean-field theory for the phases and order-parameter profiles of the inhomogeneous, extended Bose-Hubbard model (1) by generalizing earlier studies for the spinless [7, 14] and spin-1 [12] Bose-Hubbard models. In the homogeneous case, our theory leads to SF, MI, DW, and SS phases and phase diagrams, such as those of Figs. 1 (a) and (b); these are qualitatively similar to those obtained by a Gutzwiller approximation in Ref. [15]. In the inhomogeneous case, i.e., with $V_{T}>0$ in the extended Bose-Hubbard model (1), our theory lead to rich, order parameter-profiles (see, e.g., Figs. 3. 4, 5] and 7). We have also explored the Fourier-space manifestations of these profiles, the structures of the shells of SF, MI, DW, and SS phases and the associated integrated, in-trap density profiles for representative parameter values. Such shell structure has been explored for cold-atom systems that can be modelled by Bose-Hubbard models [10, 14, 20 26] but not for the extended Bose-Hubbard model.

To make a detailed comparison of our results with experiments, the parameters of the Bose-Hubbard model must be related to experimental ones [1] as follows: $\frac{U}{z t}=\frac{\sqrt{8} \pi}{4 z} \frac{a_{s}}{a} \exp \left(2 \sqrt{\frac{V_{0}}{E_{r}}}\right)$, where $E_{r}$ is the recoil energy, $V_{0}$ the strength of the lattice potential, $a_{s}\left(=5.45 \mathrm{~nm}\right.$ for $\left.{ }^{87} \mathrm{Rb}\right)$ the $s$-wave scattering coefficient, $a=\lambda / 2$ the optical lattice constant, and $\lambda=825 \mathrm{~nm}$ the wavelength of the laser used to create the optical lattice; typically $0 \leq V_{0} \leq 22 E_{r}$. If we use this experimental parametrization, we scale all the energies by $E_{r}$. [In this paper, we set $z t=1$, i.e., we measure all energies in units of $z t$. . For the extended Bose-Hubbard case, the relation of our model parameters to parameters in dipolar systems [16, 17] is not straightforward because of the long-range interactions. However, rough estimates can 
be made as follows:

$$
t=\int w^{*}\left(\mathbf{r}-\mathbf{r}_{i}\right)\left[\frac{-\hbar^{2}}{2 m} \nabla^{2}+V_{l}(\mathbf{r})\right] w\left(\mathbf{r}-\mathbf{r}_{j}\right) d^{3} r
$$

where $i$ and $j$ are nearest-neighbor sites, $w$ are Wannier functions, and $V_{l}(\mathbf{r})=\sum_{\alpha=x, y, z} V_{\alpha}^{2} \cos ^{2}\left(k_{\alpha} \alpha\right)$ is the opticallattice potential with wavevector k. Furthermore,

$$
U=U_{i i}=\int\left|w\left(\mathbf{r}-\mathbf{r}_{i}\right)\right|^{2} V_{\mathrm{int}}\left(\mathbf{r}-\mathbf{r}^{\prime}\right)\left|w\left(\mathbf{r}^{\prime}-\mathbf{r}_{i}\right)\right|^{2} d^{3} r d^{3} r^{\prime}
$$

and

$$
V=U_{<i j>}=\int\left|w\left(\mathbf{r}-\mathbf{r}_{i}\right)\right|^{2} V_{\text {int }}\left(\mathbf{r}-\mathbf{r}^{\prime}\right)\left|w\left(\mathbf{r}^{\prime}-\mathbf{r}_{j}\right)\right|^{2} d^{3} r d^{3} r^{\prime},
$$

with

$$
V_{\mathrm{int}}=D^{2} \frac{1-3 \cos ^{2} \theta}{\left|\mathbf{r}-\mathbf{r}^{\prime}\right|^{3}}+\frac{4 \pi \hbar^{2} a_{s}}{m} \delta\left(\mathbf{r}-\mathbf{r}^{\prime}\right) .
$$

Here $D$ is the dipole moment, $a_{s}$ is the $s$-wave scattering constant, and $m$ is the mass. The $s$-wave scattering constant of chromium is $\left|a\left({ }^{52} \mathrm{Cr}\right)\right|=(170 \pm 39) a_{0}$ and $\left|a\left({ }^{50} \mathrm{Cr}\right)\right|=(40 \pm 15) a_{0}$, where $a_{0}=0.053 \mathrm{~nm}$ [27].

We hope that our work will stimulate experiments designed to explore SF, MI, DW, and SS shells in dipolarcondensate systems [16, 17].

\section{ACKNOWLEDGMENTS}

We thank A. Bhatnagar, K. Rajany, and especially K. Sheshadri, S. Mukerjee and S. Bhattacharjee for discussions, and DST, CSIR, and UGC (India) for support. We would like to dedicate this paper to Professor Ulrich Eckern on the occasion of his sixtieth birthday.

[1] I. Bloch, J. Dalibard, and W. Zwerger, Rev. Mod. Phys. 80, 885 (2008).

[2] M. Lewenstein, et al., Adv. in Physics, 56243 (2007).

[3] D. Jaksch, et al., Phys. Rev. Lett. 81, 3108 (1998).

[4] M. Greiner, et al., Nature (London) 415, 39 (2002).

[5] M.P.A. Fisher, et al., Phys. Rev. B 40, 546 (1989); D.S. Rokhsar and B. G. Kotliar, Phys. Rev. B 44, 10328 (1991); W. Krauth, M. Caffarel, and J-P. Bouchaud, Phys. Rev. B 45, 3137 (1992).

[6] W. Krauth, N. Trivedi, and D. Ceperley, Phys. Rev. Lett. 67, 2307 (1991); N. Trivedi and M. Makivic, ibid. 74, 1039 (1995).

[7] K. Sheshadri, et al., Europhys. Lett. 22257 (1993).

[8] S. Wessel, et al., Phys. Rev. A, 70053615 (2004).

[9] V.A. Kashurnikov, N.V. Prokofev, and B.V. Svistunov, Phys. Rev. A, 66, 031601 (2002).

[10] S. Fölling, et al., Phys. Rev. Lett. 97, 060403 (2006).

[11] G.K. Campbell, et al., Science 313, 649 (2006).

[12] R. V. Pai, K. Sheshadri and R. Pandit, Phys. Rev. B 77, 014503 (2008).

[13] K. Sheshadri, et al., Phys. Rev. Lett. 754075 (1995).

[14] R.V. Pai, J.M. Kurdestany, K. Sheshadri, and R. Pandit, arXiv:1201.1642 (2012).

[15] D. Kovrizhin, G.V. Pai, S. Sinha, Europhys. Lett. 72, 162 (2005).

[16] J. Werner, et al., Phys. Rev. Lett. 94, 183201 (2005).

[17] K. Göral, L. Santos, M. Lewenstein, Phys. Rev. Lett. 88, 170406 (2002).

[18] A.F. Andreev and I.M. Lifshitz, Sov. Phys. JETP, 29, 1107 (1969); A.J. Leggett, Phys. Rev. Lett. 25, 1543 (1970); G. Chester, Phys. Rev. A 2, 256 (1970).

[19] E. Kim and M.H.W. Chan, Nature 427, 225 (2004).

[20] S. Bergkvist, P. Henelius, and A. Rosengren, Phys. Rev. A 70, 053601 (2004).

[21] L. Pollet, et al., Phys. Rev. A 69, 043601 (2004).

[22] B. DeMarco, et al., Phys. Rev. A 71, 063601 (2005).

[23] K. Mitra, C.J. Williams, and C. A. R. Sá de Melo, Phys. Rev. A 77, 033607 (2008). 
[24] I. B. Spielman, W. D. Phillips, and J. V. Porto, Phys. Rev. Lett. 98, 080404 (2007); ibid 100, 120402 (2008).

[25] Y. Kato, et al., Nature Physics 4, 617 (2008).

[26] T. Ozaki and T. Nikuni, J. Phys. Conference Series 150, 042158 (2009).

[27] P.O. Schmidt, et al., Phys. Rev. Lett. 91, 193201 (2003). 